\title{
Using Complete Squares Method to Optimize Replenishment Policies in a Four-Stage Supply Chain with Planned Backorders
}

\author{
M. E. Seliaman \\ Department of Information Systems, College of Computer Science and Information Technology, \\ King Faisal University, Alhsaa 3198, Saudi Arabia \\ Correspondence should be addressed to M. E. Seliaman, mseliaman@gmail.com
}

Received 1 January 2011; Revised 16 May 2011; Accepted 1 June 2011

Academic Editor: Roger Z. Ríos-Mercado

Copyright (C 2011 M. E. Seliaman. This is an open access article distributed under the Creative Commons Attribution License, which permits unrestricted use, distribution, and reproduction in any medium, provided the original work is properly cited.

\begin{abstract}
We develop a four-stage, serial supply chain inventory model with planned backorders. This supply chain model is formulated for the integer multipliers coordination mechanism, where firms at the same stage of the supply chain use the same cycle time and the cycle time at each stage is an integer multiplier of the cycle time used at the adjacent downstream stage. We develop an optimal replenishment policy using a simple algebraic method to solve the problem without the use of differential calculus.
\end{abstract}

\section{Introduction}

Recently firms realized that the global system performance and cost efficiency can be improved through closer collaboration among the chain partners and through high level of coordination of various decision processes. Supply chain inventory-distribution coordination can be achieved by coordinating the cycle time across the chain stages. The simplest way of cycle coordination is the equal cycle under which the same cycle is followed throughout the supply chain. But many supply models achieve coordination by following the integer multipliers mechanism in which the cycle time at each stage is an integer multiple of the cycle time of the adjacent downstream stage, or by integer powers of two multipliers at each stage mechanism (Khouja [1]).

In recent years numerous articles in supply chain modeling have addressed the issue of inventory coordination. Banerjee [2] introduced the concept of joint economic lot sizing problem (JELS) for the case of a single vendor and a single purchaser. Goyal and Szendrovits [3] presented a constant lot size model where the lot is produced through a fixed sequence of 
manufacturing stages, with a single setup and without interruption at each stage. Goyal [4] provided a more general model for the case of single vendor single buyer through relaxing the lot for lot policy. Goyal and Gupta [5] extensively reviewed the literature which deals with the interaction between a buyer and vendor. $\mathrm{Lu}$ [6] developed a single vendor multibuyer integrated inventory model. Goyal [7] revisited the single-vendor single-buyer where he relaxed the constraint of equal sized shipments of Goyal [4] and suggested that the shipment size should grow geometrically. Hoque and Goyal [8] extended the idea of producing a single product in a multistage serial production system with equal and unequal sized batch shipments between stages. Khouja [1] considered the case of a three-stage nonserial supply chain and developed the model to deal with three inventory coordination mechanisms between the chain members. Ben-daya and Al-Nassar [9] relaxed the assumption of Khouja [1] regarding the completion of the whole production lot before making shipments out of it and assumed that equal sized shipments take place as soon as they are produced and there is no need to wait until a whole lot is produced.

The use of differential calculus to model the integrated production-inventory systems is common in the area of operational research. However, several researchers focused on algebraic methods for the optimization of these types of systems. Cárdenas-Barrón [10] used algebraic procedure to the EPQ formula taking shortages into consideration within the case of only one backlog cost per unit and time unit. Cárdenas-Barrón [11] used an algebraic approach to optimize the supply chain model formulated for the simplest inventory coordination mechanism presented by Khouja [1]. Chung and Wee [12] considered an integrated three-stage inventory system with backorders. They formulated the problem to derive the replenishment policies with four decision variables algebraically. Wee and Chung [13] also used a simple algebraic method to solve the economic lot size of the integrated-buyer inventory problem. Chiu [14] presented a simple algebraic method to demonstrate that the lot size solution and the optimal production-inventory cost of an imperfect EMQ model can be derived without derivatives.

This paper extends the existing models that use algebraic methods to optimize inventory decisions in multistage supply chains. It contributes by extending the well-known and popular EOQ deterministic model which is widely used for inventory management in practice as well as in research in spite of its restrictive assumptions Pentico and Drake [15]. Hence, this work contributes in the theory and solution methodology. Moreover, the recursive structures we used to drive the optimal replenishment policy can be easily used for further purposes and extensions. One example is the use of our approach to develop the scheme for sharing the benefits of coordination among the supply chain partners. In this regard, the model can form the base for the coordination negotiation.

The remainder of this paper is organized as follows. The next section presents the notation and assumptions made in the model. Section 3 describes the development of the model. A numerical example is presented in Section 4. Finally, Section 5 contains some concluding remarks.

\section{Notation and Assumptions}

The following notations are used in developing the model:

$T=$ basic cycle time, cycle time at the end retailer,

$T_{i}=$ cycle time at stage $i$,

$S_{i}=$ setup cost at stage $i$, 
$K_{i}=$ integer multiplier at stage $i$,

$h_{i}=$ inventory holding cost at stage $i$,

$D=$ the demand rate at the end stage,

$P_{i}=$ production rate at stage $i$,

$\pi=$ backordering cost per unit per unit time.

Assumptions for the multi-stage supply chain production-inventory model are as follows.

(a) A single product is produced and distributed through a four-stage serial, supply chain.

(b) Shortages are allowed for the end retailer.

(c) Replenishment is instantaneous.

(d) Production rates and demand rate are deterministic and uniform.

(e) A lot produced at stage is sent in equal shipments to the downstream stage.

(f) The entire supply chain optimization is acceptable for all partners in the chain.

(g) Complete information sharing policy is adopted.

(h) Cycle time at each stage is an integer multiplier of the cycle time used at the adjacent downstream stage.

\section{The Model}

We consider a four-stage, serial supply chain. This supply chain model is formulated for the integer multipliers coordination mechanism, where the cycle time at each stage is an integer multiplier of the cycle time used at the adjacent downstream stage. Shortages are allowed for the end retailer. The retailer's cost consists of the inventory holding cost, the shortage cost, and the setup cost. In this case as we can see, from Figure 1, that the time-weighted total cost for the retailer is given by

$$
T C_{4}=h_{4} \frac{T D_{4}}{2}-T_{S} D h_{4}+h_{4} \frac{T_{S}^{2} D}{2 T}+\pi \frac{T_{S}^{2} D}{2 T}+\frac{S_{4}}{T} .
$$

The inventory profiles at the remaining upstream stages is depicted by Figure 2. As depicted in Figure 2 the inventory holding at each stage, except for the final stage (the end retailer at stage 4), is made of two parts: the first one is the carrying inventory of raw materials as they are being converted into finished products during the production portion of the cycle. The second part is the carrying inventory of the finished products during the nonproduction 
portion of the cycle (Khouja [1]). Therefore, the total time-weighted cost for each of the third, second, and first stages is represented by following equations, respectively,

$$
\begin{gathered}
T C_{3}=K_{3} \frac{T D^{2}}{2 P_{3}}\left(h_{2}+h_{3}\right)+\left(K_{3}-1\right) \frac{T D}{2} h_{3}+\frac{S_{3}}{K_{3} T} \\
T C_{2}=K_{2} K_{3} \frac{T D^{2}}{2 P_{2}}\left(h_{1}+h_{2}\right)+K_{3}\left(K_{2}-1\right) \frac{T D}{2} h_{2}+\frac{S_{2}}{K_{2} K_{3} T}, \\
T C_{1}=K_{1} K_{2} K_{3} \frac{T D^{2}}{2 P_{1}}\left(h_{0}+h_{1}\right)+K_{2} K_{3}\left(K_{1}-1\right) \frac{T D}{2} h_{1}+\frac{S_{1}}{K_{1} K_{2} K_{3} T} .
\end{gathered}
$$

The entire supply time-weighted cost is

$$
\begin{aligned}
T C= & \sum_{i, j} T C_{i, j} \\
= & \frac{T}{2}\left\{D h_{4}-D h_{3}+K_{3}\left[\frac{D^{2}}{P_{3}}\left(h_{2}+h_{3}\right)+D h_{3}-D h_{2}\right]+K_{3} K_{2}\left[\frac{D^{2}}{P_{2}}\left(h_{1}+h_{2}\right)+D h_{2}-D h_{1}\right]\right. \\
& \left.+K_{3} K_{2} K_{1}\left[\frac{D^{2}}{P_{1}}\left(h_{0}+h_{1}\right)+D h_{1}\right]\right\} \\
+ & \frac{1}{T}\left\{S_{4}+\frac{S_{3}}{K_{3}}+\frac{S_{2}}{K_{2} K_{3}}+\frac{S_{1}}{K_{1} K_{2} K_{3}}\right\} \\
+ & \frac{D\left(h_{4}+\pi\right)}{2 T}\left\{T_{S}^{2}-\frac{2 T_{S} h_{4} T}{h_{4}+\pi}\right\} .
\end{aligned}
$$

Now by using the method of completing square with respect to $T_{S}$ as in Cárdenas-Barrón [11], (3.3) can be rewritten as

$$
\begin{aligned}
T C= & \frac{T}{2}\left\{D h_{4}-D h_{3}-\frac{D h_{4}^{2}}{h_{4}+\pi}+K_{3}\left[\frac{D^{2}}{P_{3}}\left(h_{2}+h_{3}\right)+D h_{3}-D h_{2}\right]\right. \\
& \left.+K_{3} K_{2}\left[\frac{D^{2}}{P_{2}}\left(h_{1}+h_{2}\right)+D h_{2}-D h_{1}\right]+K_{3} K_{2} K_{1}\left[\frac{D^{2}}{P_{1}}\left(h_{0}+h_{1}\right)+D h_{1}\right]\right\} \\
+ & \frac{1}{T}\left\{S_{4}+\frac{S_{3}}{K_{3}}+\frac{S_{2}}{K_{2} K_{3}}+\frac{S_{1}}{K_{1} K_{2} K_{3}}\right\} \\
+ & \frac{D\left(h_{4}+\pi\right)}{2 T}\left\{T_{S}-\frac{h_{4} T}{h_{4}+\pi}\right\}^{2} .
\end{aligned}
$$

Rewriting (3.4), one has

$$
T C=T Y+\frac{W}{T}+\frac{D\left(h_{4}+\pi\right)}{2 T}\left\{T_{S}-\frac{h_{4} T}{h_{4}+\pi}\right\}^{2},
$$




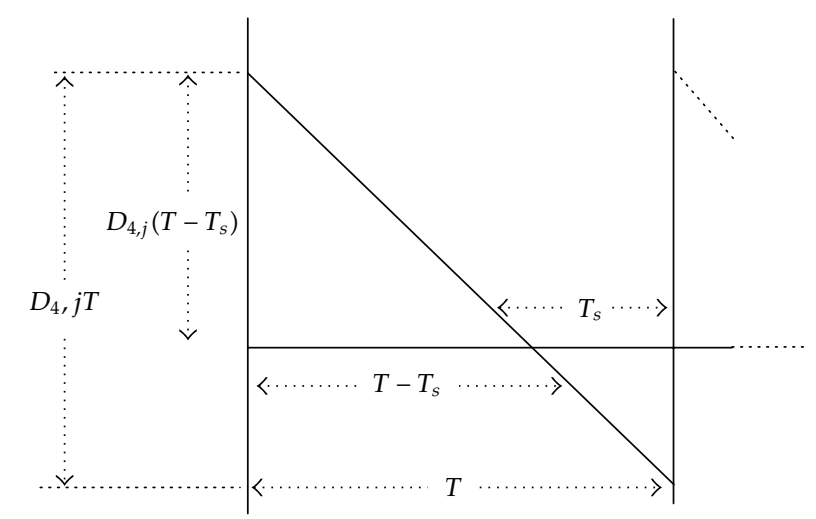

Figure 1: The inventory level at the end retailer.

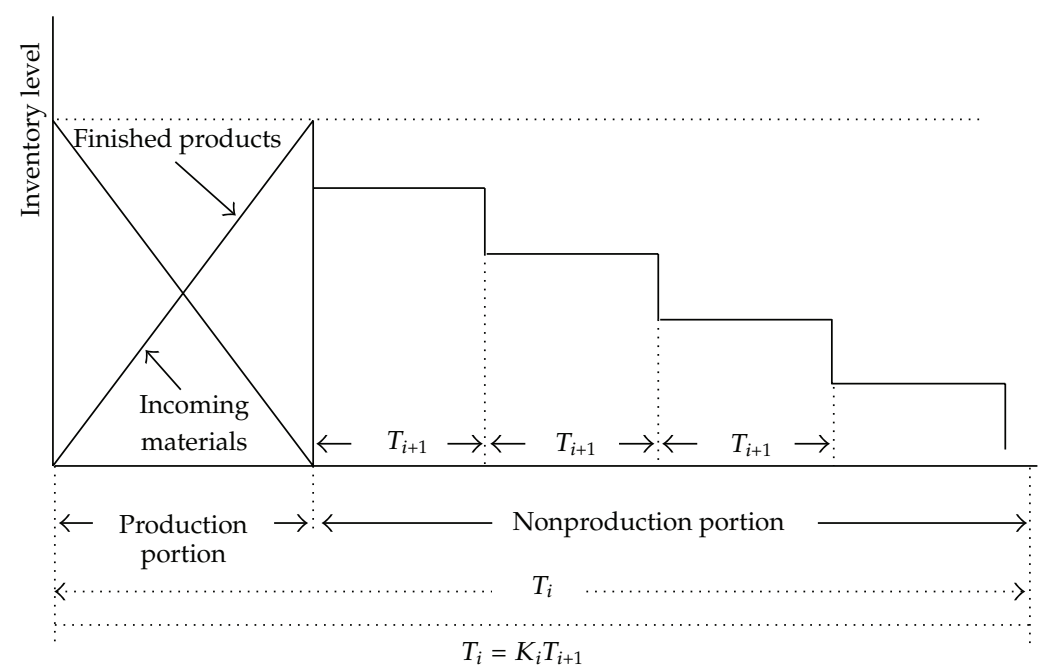

Figure 2: The inventory holdings at firms other than the end retailer.

where $Y=\left(K_{3} \psi_{3}+\alpha_{3}\right) / 2, \alpha_{3}=D h_{4}-D h_{3}-\left(h_{4}^{2} / h_{4}+\pi\right), \psi_{3}=K_{2} \psi_{2}+\alpha_{2}, \alpha_{2}=\left(D^{2} / P_{3}\right)\left(h_{2}+h_{3}\right)+$ $D h_{3}-D h_{2}, \psi_{2}=K_{1} \psi_{1}+\alpha_{1}, \alpha_{1}=\left(D^{2} / P_{2}\right)\left(h_{1}+h_{2}\right)+D h_{2}-D h_{1}, \psi_{1}=\left(D^{2} / P_{1}\right)\left(h_{0}+h_{1}\right)+D h_{1}$, $W=\left(S_{4}+\left(\varphi_{3} / K_{3}\right)\right), \varphi_{3}=\left(S_{3}+\left(\varphi_{2} / K_{2}\right)\right), \varphi_{2}=\left(S_{2}+\left(S_{1} / K_{1}\right)\right)$.

Now applying the algebraic procedure proposed by Cárdenas-Barrón [10], the annual total cost for the entire supply chain in (3.5) can be represented by factorizing the term $1 / T$ and completing the perfect square, and one has

$$
T C=\frac{1}{T}\left(T^{2} Y-2 T \sqrt{Y W}+W+2 T \sqrt{Y W}\right)+\frac{D\left(h_{4}+\pi\right)}{2 T}\left\{T_{S}-\frac{h_{4} T}{h_{4}+\pi}\right\} .
$$

Factorizing the perfect squared trinomial in a squared binomial we obtain

$$
T C=\frac{1}{T}(T \sqrt{Y}-\sqrt{W})^{2}+\frac{D\left(h_{4}+\pi\right)}{2 T}\left\{T_{S}-\frac{h_{4} T}{h_{4}+\pi}\right\}+2 \sqrt{Y W}
$$


It is worthy pointing out that (3.7) reaches minimum with respect to $T$ and $T_{S}$ when setting

$$
(T \sqrt{Y}-\sqrt{W})^{2}=0, \quad\left\{T_{S}-\frac{h_{4} T}{h_{4}+\pi}\right\}=0 .
$$

Hence, the optimal basic cycle time $T^{*}$ is

$$
T^{*}=\sqrt{\frac{W}{Y}}
$$

and the optimal allowable stocking out time $T_{S}^{*}$ is

$$
T_{S}^{*}=\frac{h_{4}}{h_{4}+\pi} T^{*}
$$

Substituting (3.9) and (3.10) into (3.7), the minimum value for the annual total cost for the entire supply chain is

$$
T C=2 \sqrt{Y W}
$$

The optimal basic cycle time $T^{*}$ is a function of the integer multipliers $\left(K_{3}, K_{2}, K_{1}\right)$. We use the method of perfect square to drive the optimal values of these integer multipliers iteratively. Substituting for $Y$ and $W$ into (3.11) we get

$$
\begin{aligned}
T C & =\sqrt{2}\left\{\left(K_{3} \psi_{3}+\alpha_{3}\right)\left(S_{4}+\frac{\varphi_{3}}{K_{3}}\right)\right\}^{1 / 2} \\
& =\sqrt{2}\left\{\frac{1}{K_{3}}\left[K_{3} \sqrt{\psi_{3} S_{4}}-\sqrt{\alpha_{3} \varphi_{3}}\right]^{2}+\left[\sqrt{\psi_{3} \varphi_{3}}+\sqrt{\alpha_{3} S_{3}}\right]^{2}\right\}^{1 / 2} .
\end{aligned}
$$

From (3.12) setting

$$
\left[K_{3} \sqrt{\psi_{3} S_{4}}-\sqrt{\alpha_{3} \varphi_{3}}\right]^{2}=0
$$

the optimal value of integer multiplier $K_{3}$ is derived as follows:

$$
K^{*}{ }_{3}=\sqrt{\frac{\alpha_{3} \varphi_{3}}{\psi_{3} S_{4}}} .
$$

Since the value of $K_{3}$ is a positive integer, the following condition must be satisfied:

$$
\left(K^{*}{ }_{3}\right) \cdot\left(K^{*}{ }_{3}-1\right) \leq\left(K^{*}\right)^{2} \leq\left(K^{*}{ }_{3}\right) \cdot\left(K^{*}{ }_{3}+1\right) .
$$


To drive the optimal value of integer multiplier $K_{2}$, we can rewrite the term $\sqrt{\psi_{3} \varphi_{3}}$ in (3.12) as follows:

$$
\sqrt{\psi_{3} \varphi_{3}}=\left\{\frac{1}{K_{2}}\left[K_{2} \sqrt{\psi_{2} S_{3}}-\sqrt{\alpha_{2} \varphi_{2}}\right]^{2}+\left[\sqrt{\psi_{2} \varphi_{2}}+\sqrt{\alpha_{2} S_{3}}\right]^{2}\right\} .
$$

From (3.16), setting

$$
\left[K_{2} \sqrt{\psi_{2} S_{3}}-\sqrt{\alpha_{2} \varphi_{2}}\right]^{2}=0
$$

And the optimal value of the integer multiplier is derived as follows:

$$
K_{2}^{*}=\sqrt{\frac{\alpha_{2} \varphi_{2}}{\psi_{2} S_{3}}}
$$

Since the value of $K_{2}$ is a positive integer, the following condition must be satisfied:

$$
\left(K_{2}^{*}\right) \cdot\left(K_{2}^{*}-1\right) \leq\left(K_{2}^{*}\right)^{2} \leq\left(K_{2}^{*}\right) \cdot\left(K_{2}^{*}+1\right) .
$$

Similarly, the optimal value of the integer multiplier $K_{1}$ is reached:

$$
K^{*}=\sqrt{\frac{\alpha_{1} S_{1}}{\psi_{1} S_{2}}}=\sqrt{\frac{\left[D\left(h_{1}+h_{2}\right) / P_{2}+h_{2}-h_{1}\right] S_{1}}{\left[D\left(h_{0}+h_{1}\right) / P_{1}+h_{1}\right] S_{2}}} .
$$

Since the value of $K_{1}$ is a positive integer, the following condition must be satisfied:

$$
\left(K^{*}{ }_{1}\right) \cdot\left(K^{*}{ }_{1}-1\right) \leq\left(K^{*}{ }_{1}\right)^{2} \leq\left(K^{*}{ }_{1}\right) \cdot\left(K^{*}{ }_{1}+1\right) .
$$

Now, we can use $K^{*}{ }_{1}$ to find $K^{*}{ }_{2}$ which can be used to find $K^{*}{ }_{3}$.

\section{Numerical Example}

In this section, we consider an example of a four-stage supply chain consisting of a supplier, a manufacturer, a distributor, and a retailer. This example was originated by Goyal [7] for only two stages and was later extended by Chung and Wee [12] to include a distributor stage. To illustrate the model presented here in this paper, we added a supplier stage to the example. A typical structure for such supply chain system is depicted in Figure 3, and the relevant data is shown in Table 1. In addition to this data, backordering cost per unit per unit time is assumed 20. It is also assumed that holding cost for the supplier's supplier is $h_{0}=0.5$.

By applying the developed algebraic solution procedure in Section 3, the results of this example are presented in Table 2. The optimal production lot size for the supplier is 816.07 which will be delivered to the manufacturer in two deliveries. The optimal production lot 


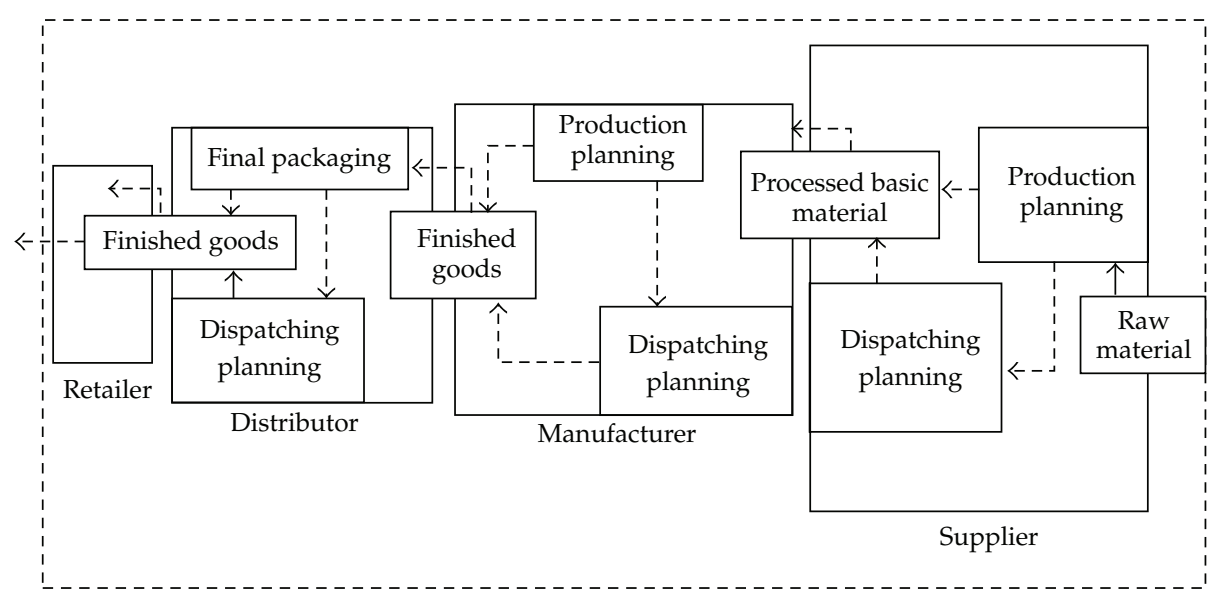

Figure 3: Four stage supply chain example.

Table 1: Data for the example supply chain.

\begin{tabular}{ccccc}
\hline$i$ & $P_{i}$ & $D$ & $h_{i}$ & $S_{i}$ \\
\hline 1 & 4000 & 1000 & 2 & 650 \\
2 & 3200 & 1000 & 4 & 400 \\
3 & 2500 & 1000 & 4.2 & 40 \\
4 & - & 1000 & 5 & 25 \\
\hline
\end{tabular}

Table 2: The Example solution.

\begin{tabular}{lc}
\hline Parameter & Solution \\
\hline$K_{1}$ & 2 \\
$K_{2}$ & 3 \\
$K_{3}$ & 1 \\
$T$ & 0.136 \\
$T_{S}$ & 0.019 \\
$T C$ & 5409.39 \\
\hline
\end{tabular}

size for the manufacturer is 408.04 which will be delivered to the distributor in three batches. However, the distributor and the retailer will share the same cycle length and the optimal batch size delivered by the distributor to the retailer is 136.01 .

\section{Conclusions}

In this study, we develop a four-stage, serial supply chain inventory model with planned backorders. This chain is consisted of a supplier, a manufacturer, distributor, and an end retailer. Production and inventory decisions are made at the supplier, manufacturer; and distributor levels. The production and demand rates are assumed finite. In addition backorders are permitted for unmet demand at the retailer. The problem is to coordinate production and inventory decisions across the supply chain so that the total cost of the system 
is minimized. This supply chain model is formulated for the integer multipliers coordination mechanism, where firms at the same stage of the supply chain use the same cycle time and the cycle time at each stage is an integer multiplier of the cycle time used at the adjacent downstream stage. We develop an optimal replenishment policy using a simple algebraic method to solve the problem without the use of differential calculus.

One clear limitation of the developed model is the assumption of known deterministic demand. Considering a real-world case and relaxing the restrictive model assumptions are a good direction for further research.

\section{Acknowledgments}

The author would like to acknowledge the support for this research provided by King Faisal University. Acknowledgment is also due to the editor and reviewers for their comments and feedback on this paper.

\section{References}

[1] M. Khouja, "Optimizing inventory decisions in a multi-stage multi-customer supply chain," Transportation Research Part E: Logistics and Transportation Review, vol. 39, no. 3, pp. 193-208, 2003.

[2] A. Banerjee, "A joint economic-lot-size model for purchaser and vendor," Decision Sciences, vol. 17, no. 3, pp. 292-311, 1986.

[3] S. K. Goyal and A. Z. Szendrovits, "A constant lot size model with equal and unequal sized batch shipments between production stages," Engineering Costs and Production Economics, vol. 10, no. 1, pp. 203-210, 1986.

[4] S. K. Goyal, "A one-vendor multi-buyer integrated inventory model: a comment," European Journal of Operational Research, vol. 82, no. 1, pp. 209-210, 1995.

[5] S. K. Goyal and Y. P. Gupta, "Integrated inventory models: the buyer-vendor coordination," European Journal of Operational Research, vol. 41, no. 3, pp. 261-269, 1989.

[6] L. Lu, "A one-vendor multi-buyer integrated inventory model," European Journal of Operational Research, vol. 81, no. 2, pp. 312-323, 1995.

[7] S. K. Goyal, "A joint economic-lot-size model for a purchaser and a comment," Decision Sciences, vol. 19, no. 1, pp. 236-241, 1988.

[8] M. A. Hoque and S. K. Goyal, "An optimal policy for a single-vendor single-buyer integrated production-inventory system with capacity constraint of the transport equipment," International Journal of Production Economics, vol. 65, no. 3, pp. 305-315, 2000.

[9] M. Ben-daya and A. Al-Nassar, "Integrated multi-stage multi-customer supply chain," Production Planning E Control the Management of Operations, vol. 19, no. 2, pp. 97-104, 2008.

[10] L. E. Cárdenas-Barrón, “Optimizing inventory decisions in a multi-stage multi-customer supply chain: a note," Transportation Research, Part E: Logistics and Transportation Review, vol. 43, no. 5, pp. 647-654, 2007.

[11] L. E. Cárdenas-Barrón, “The economic production quantity (EPQ) with shortage derived algebraically," International Journal of Production Economics, vol. 70, no. 3, pp. 289-292, 2001.

[12] C. J. Chung and H. M. Wee, "Optimizing the economic lot size of a three-stage supply chain with backordering derived without derivatives," European Journal of Operational Research, vol. 183, no. 2, pp. 933-943, 2007.

[13] H. M. Wee and C. J. Chung, "A note on the economic lot size of the integrated vendor-buyer inventory system derived without derivatives," European Journal of Operational Research, vol. 177, no. 2, pp. 12891293, 2006.

[14] S. W. Chiu, "Production lot size problem with failure in repair and backlogging derived without derivatives," European Journal of Operational Research, vol. 188, no. 2, pp. 610-615, 2008.

[15] D. W. Pentico and M. J. Drake, "A survey of deterministic models for the EOQ and EPQ with partial backordering," European Journal of Operational Research, vol. 214, no. 2, pp. 179-198, 2010. 


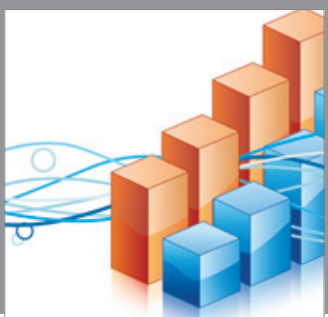

Advances in

Operations Research

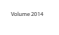

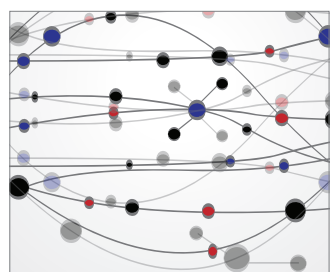

\section{The Scientific} World Journal
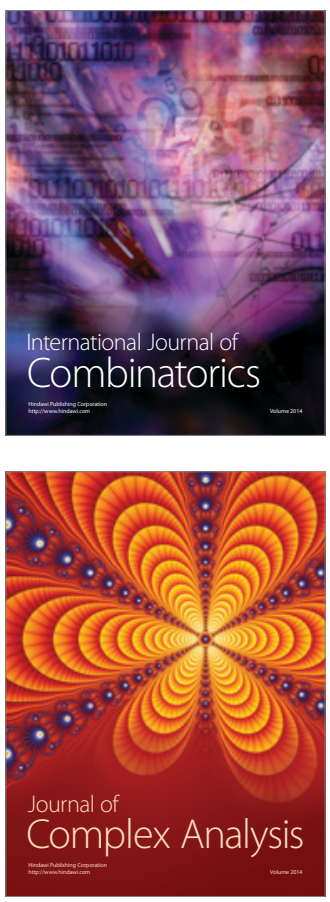

International Journal of

Mathematics and

Mathematical

Sciences
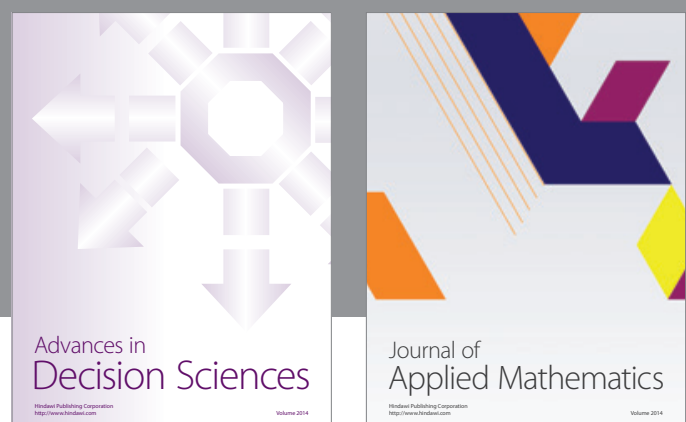

Journal of

Applied Mathematics
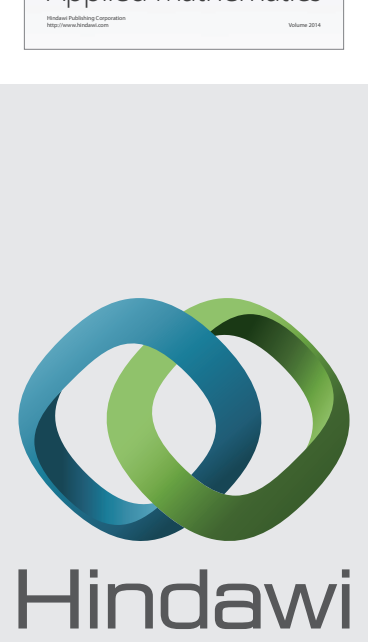

Submit your manuscripts at http://www.hindawi.com
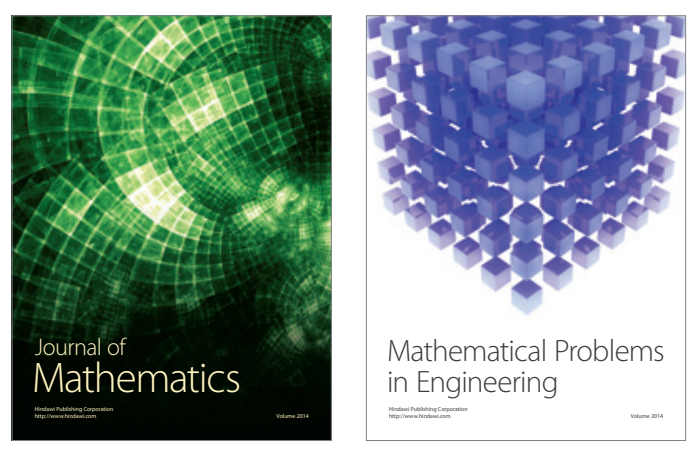

Mathematical Problems in Engineering
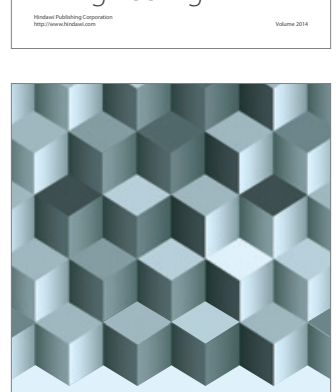

Journal of

Function Spaces
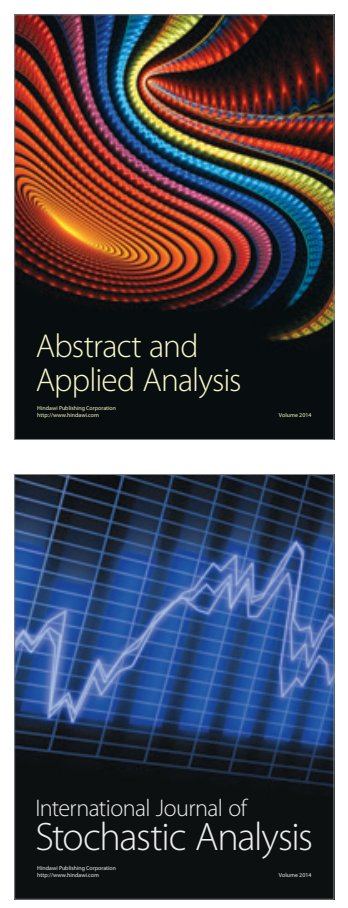

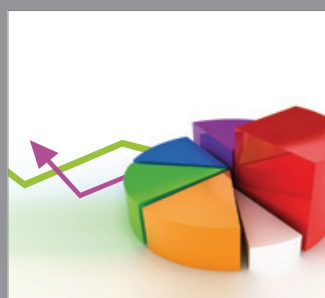

ournal of

Probability and Statistics

Promensencen
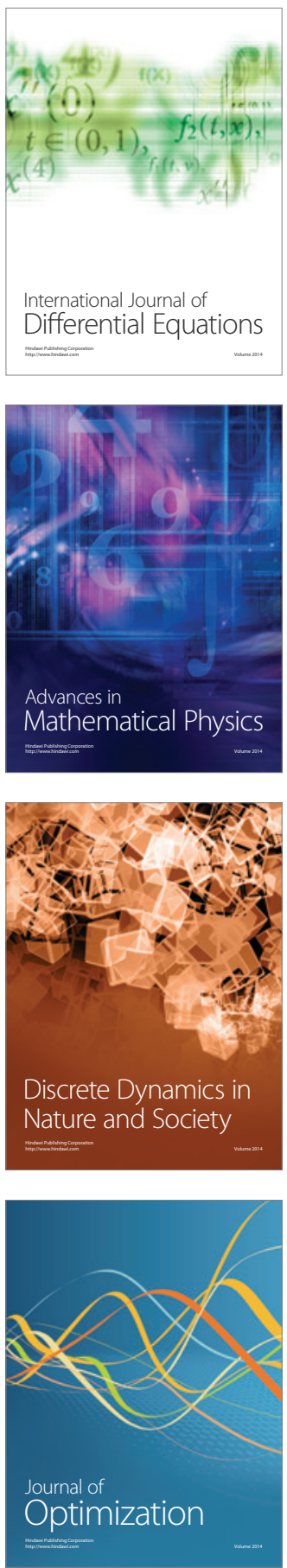